\title{
DEEP INTERARYTENOID NOTCH IN YOUNG CHILDREN MANAGED WITH SYSTEMATIC THICKENER WEAN AND INJECTION LARYNGOPLASTY
}

\begin{abstract}
A thesis submitted to the University of Arizona College of Medicine - Phoenix in partial fulfillment of the requirements for the Degree of Doctor of Medicine
\end{abstract}

Usmaan Basharat

Class of 2019

Mentor: Dana Williams, MD 


\section{Acknowledgements}

- University of Arizona College of Medicine - Phoenix, Scholarly Project

- Cortney Holmes, Clinical Data Coordinator

- Jessica Smith, Pediatric NP Coordinator

- Annette Martinez, MA, Aerodigestive Clinic Coordinator

Funding is provided by the Connell Family Aerodigestive Research Fund 


\begin{abstract}
Objectives: Deep interarytenoid notch (DIN) is a congenital variation of the larynx often associated with dysphagia and aspiration (DA) in young children. Feeding therapy with thickeners and surgical management with injection larygoplasty (IL) are used with various efficacies. Thickeners address the functional domain and IL addresses the anatomical domain of treatment. Our objective was to evaluate DIN patients managed with both interventions. Methods: We conducted a retrospective pilot descriptive study of DIN patients with DA aged 13 years receiving thickeners and IL. Patients received a systematic weekly reduction of thickeners based on clinical signs and symptoms of DA. The outcomes were assessed by the rate of thickener level reduction and DA-related sign/symptom frequency achieved at 6 months post-treatment.
\end{abstract}

Results: Thirteen patients with DIN associated DA were analyzed. Thickener scores improved from an average of 5.76 (3/4 honey) to 2.15 (thin) ( $p=0.001)$. DA-related signs/symptoms frequency improved from an average of 3.3 to $0.84(p=0.05)$.

Conclusions: These findings suggest that treatment of DIN associated DA with a combination of thickeners and IL results in significant clinical improvements in young children.

Keywords: deep interarytenoid notch; injection laryngoplasty; thickener; dysphagia; aspiration; laryngeal cleft 


\section{Table of Contents}

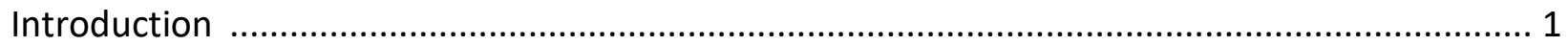

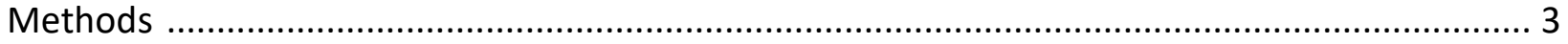

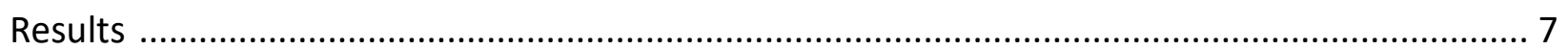

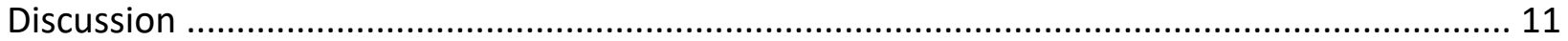

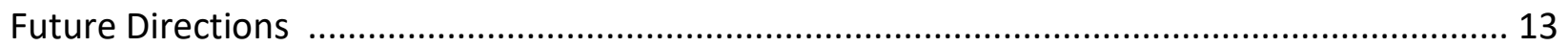

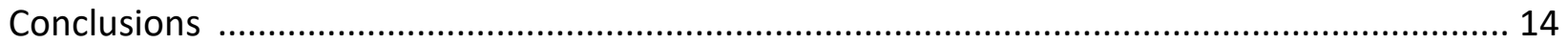

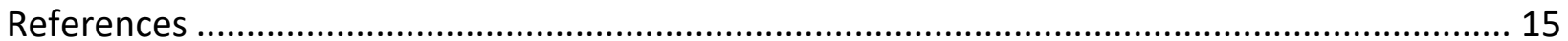




\section{List of Figures and Tables:}

Table 1: Thickener consistency/liquid viscosity scores

Table 2: Dysphagia and aspiration questionnaire

Table 3: Patient demographics

Figure 1: Thickener levels before and after therapy 


\section{Introduction}

The incidence of dysphagia has been increasing in children, and a portion of these patients are found to have a laryngeal cleft on further evaluation[1]. Type 1 laryngeal clefts (LC1) are the most common and mild form of this defect, as described by the Benjamin and Inglis classification system[2]. LC1s are defined as defects in the interarytenoid area extending no further inferiorly than the true vocal cords. The defect is in the interarytenoid musculature, with or without absence of the overlying mucosa. A normal interarytenoid notch height (IANH) in otherwise healthy young children is greater than $3 \mathrm{~mm}$, and on average $3.5 \mathrm{~mm}[3]$. A subclassification of LC1s has emerged more recently which includes shorter interarytenoid notch heights (less than $3 \mathrm{~mm}$ above the glottis) with normal musculature[4], which we term deep interarytenoid notches (DIN)[5]. Although DINs have not been described much in the literature, our institutional experience is that they are frequently found in children presenting with DA[6] to an aerodigestive multidisciplinary clinic. In 2013, PCH ADC found that 24\% (114/471) of young patients presenting with dysphagia and aspiration (DA) were diagnosed with DIN. DINs are a normal congenital variant and their significance in the symptomatology of patients is unclear.

The presentation of LC1 commonly involves a combination of both airway and feeding difficulties [7], including aspiration/penetration, recurrent pneumonias, respiratory distress, stridor, dysphagia, feeding problems, and failure to thrive. Penetration is defined as the passage of material into the larynx but not below the level of the vocal folds, whereas aspiration is when material enters the airway below the level of the vocal folds [8]. Either can occur with LC1/DIN. Additionally, laryngeal cleft patients with aspiration/penetration are also found to have swallow dysfunction across all phases of swallow (oral phase, pharyngeal phase, etc.)[9].

Initial workup for these patients includes modified barium swallow (MBS) evaluation, where a speech and language pathologist (SLP) administers various consistencies of food containing radiolabeled tracer seen under fluoroscopy. When MBS is inconclusive, patients can also undergo functional endoscopic evaluation of swallowing (FEES)[10]. The abnormal MBS/FEES 
findings coupled with the presenting signs and symptoms often leads to direct laryngoscopy, the gold-standard diagnostic test for laryngeal clefts/DINs[2,11]. These patients can also have coincident pathologies contributing to their symptoms including laryngomalacia, gastroesophageal reflux disease (GERD), eosinophilic esophagitis (EOE), tracheobronchomalacia, reactive airway disease (RAD), and oral/motor delays[4]. Accordingly, a multidisciplinary approach in the evaluation and treatment of patients presenting with DA has gained traction[12].

Treatment for these clefts can involve surgical intervention to address the anatomical defect or functional therapy utilizing feeding therapy with dysphagia management. These patients can also be treated medically for comorbid conditions, such as GERD and RAD, to help alleviate aerodigestive inflammation contributing to DA and to improve swallowing function[13,14]. One method of surgical intervention consists of injection laryngoplasty (IL), which has become popular lately due to the relative safety and effectiveness of the procedure $[15,16,17,18]$. IL involves injecting an aqueous gel into the interarytenoid space to provide temporary bulk to the interarytenoid area. Feeding therapy with dysphagia management includes manipulating the bolus size and flow via drinking utensil changes, thickened liquids, and addressing oral phase deficits which might contribute to pharyngeal phase dysphagia[19]. Many studies have utilized thickeners in the management of laryngeal cleft associated DA[7,17,18,20,21,22].

We present an approach involving both IL and thickened liquids aimed at rapid thickener reduction and sign/symptom improvement. Many of our patients are also on concomitant medical therapy for comorbid disease. 


\section{Methods}

We performed a descriptive pilot study through retrospective chart review of pediatric patients aged 1-3 years old who presented to the PCH ADC between January 2015 and September 2016 with DA and were diagnosed with DIN. PCH ADC uses a multidisciplinary approach for such patients. They routinely undergo subspecialty clinic evaluation (otolaryngology, gastroenterology, pulmonology, and SLP), triple endoscopy as indicated (direct laryngoscopy, esophagogastroduodenoscopy (EGD), and bronchoscopy), and feeding evaluation (using MBS and sometimes FEES). Decision for IL is made at the time of diagnosis during endoscopy procedures and is completed at that time or scheduled as a separate procedure. This decision is made by the otolaryngologist based on history, clinical symptoms, and other anatomical findings, including depth of DIN as observed by direct laryngoscopy.

We examined the clinical outcomes of the patients who were treated with both IL and PCH's proprietary thickener weaning protocol (TWP) (detailed below). Sixty one charts with the diagnosis of DIN were reviewed. Inclusion criteria included age 1-3 years at the time of diagnosis of DIN, IL, and having started TWP within 6 months of IL. Patients were excluded if they had any history of severe neurodevelopmental delay, prior esophageal surgery, endoscopic repair of DIN, or if the time interval between IL and start of TWP was greater than 6 months.

The primary outcome was the rate of thickener level reduction over the 6 months of TWP. Terminology used to describe liquid viscosity is consistent with the National Dysphagia Diet[23]. These include spoon-thick (>1750 centipoise (cP)), honey-like (351-1750 cP), nectar like (51-350 $\mathrm{cP})$, and thin (1-50 cP). Our institution also found the need to describe 'half' and 'three quarter' recipes when using SimplyThick (SimplyThick, LLC) gel and/or infant cereal to thicken liquids, because children often showed DA when transitioning from honey-like to nectar-like liquids. Use of a more gradual thickener wean is well tolerated by children and many are able to advance to a thin-fluid diet[24]. To obtain measurable and quantifiable information for the purposes of our study, our institution developed a numerical scale to quantify thickened liquids and help describe severity of dysphagia (table 1). The scores range from ultrathin (1) to pudding 
consistency (9). Higher scores indicate thicker liquids and more severe dysphagia. The SLP's diet recommendation were scored according to the aforementioned table based on the thinnest consistency the patient was able to tolerate without experiencing aspiration/penetration.

The secondary outcome measure was the reduction of thickener level (clinically) and of DArelated signs/symptoms over the 6 months of TWP. This included pulmonary and gastrointestinal signs/symptoms thought to be manifestations of the DIN. A total of 17 signs and symptoms (12 gastrointestinal and 5 pulmonary) were scored using a questionnaire where frequency was reported on a numerical scale (0-4) based on parents' recall (table 2). Baseline thickener levels and signs/symptoms were compared to the corresponding values at 6 months into TWP therapy (or sooner if the patient was able to be weaned completely off the thickeners).

The Wilcoxon signed rank test was used to compare pre-treatment and post-treatment results of the primary and secondary outcomes, and a p-value of $<0.05$ was considered statistically significant. The sub-stratification groups were compared using the Wilcoxon rank sum test, again using a p-value of $<0.05$ as statistically significant. 
Table 1: Thickener consistency/liquid viscosity scores

\begin{tabular}{|l|l|c|}
\hline \multicolumn{1}{|c|}{$\begin{array}{c}\text { MBS Thickness } \\
\text { Recommendation }\end{array}$} & \multicolumn{1}{|c|}{ Description } & 1 \\
\hline Ultrathin & Equal to water, breastmilk & 2 \\
\hline Thin & Equal to milk or formula & 3 \\
\hline $1 / 2$ nectar & $\begin{array}{l}1 \text { pack of Simply Thick nectar gel in } 8 \text { fluid ounces or } \\
1 \text { teaspoon of infant cereal per } 1 \text { fluid ounce }\end{array}$ & 4 \\
\hline $3 / 4$ nectar & $\begin{array}{l}1 \text { pack of Simply Thick nectar gel in } 6 \text { fluid ounces or } \\
1.5 \text { teaspoons of infant cereal per } 1 \text { fluid ounce }\end{array}$ & 5 \\
\hline Nectar & $\begin{array}{l}1 \text { pack of Simply Thick nectar gel in } 4 \text { fluid ounces or } \\
2 \text { teaspoons of infant cereal per } 1 \text { fluid ounce }\end{array}$ & 6 \\
\hline $3 / 4$ honey & $\begin{array}{l}1 \text { pack of Simply Thick honey gel in } 6 \text { fluid ounces or } \\
2.5 \text { teaspoons of infant cereal per } 1 \text { fluid ounce }\end{array}$ & 7 \\
\hline Honey & $\begin{array}{l}1 \text { pack of Simply Thick honey gel in } 4 \text { fluid ounces or } \\
3 \text { teaspoons of infant cereal per } 1 \text { fluid ounce }\end{array}$ & 9 \\
\hline Double honey/ & 2 packs of Simply Thick honey gel in 8 fluid ounces or \\
\hline Pudding & 3 packs of Simply Thick honey gel in 4 fluid ounces & \\
\hline
\end{tabular}


Table 2: Dysphagia and aspiration questionnaire

How often does your child have the following signs/symptoms?

\begin{tabular}{|c|c|c|c|c|c|}
\hline Cough & 0 & 1 & $\underline{2}$ & 3 & 4 \\
\hline Cough with food & 0 & 1 & 2 & 3 & 4 \\
\hline Cough with liquid & 0 & 1 & 2 & 3 & 4 \\
\hline Night time cough & 0 & 1 & 2 & 3 & 4 \\
\hline Nasal Congestion & 0 & 1 & 2 & 3 & 4 \\
\hline Chest congestion & 0 & 1 & 2 & 3 & 4 \\
\hline Wheezing & 0 & 1 & 2 & 3 & 4 \\
\hline Choke with food & 0 & 1 & 2 & 3 & 4 \\
\hline Choke with liquid & 0 & 1 & 2 & 3 & 4 \\
\hline Choke Without feed & 0 & 1 & 2 & 3 & 4 \\
\hline Gag with food & 0 & 1 & 2 & 3 & 4 \\
\hline Gag with liquid & 0 & 1 & 2 & 3 & 4 \\
\hline Gag Without feed & 0 & 1 & 2 & 3 & 4 \\
\hline Vomit & 0 & 1 & 2 & 3 & 4 \\
\hline Feeding refusal & 0 & 1 & 2 & 3 & 4 \\
\hline Wet voice & 0 & 1 & 2 & 3 & 4 \\
\hline Hoarse voice & 0 & 1 & 2 & 3 & 4 \\
\hline
\end{tabular}

(0-never, 1-monthly, 2- weekly 3-daily, 4-multiple times per day) 
Thickener wean protocol (TWP):

Phoenix Children's Hospital TWP[4] methodology was developed to treat patients with persistent dysphagia and aspiration who remain dependent on thickened liquids to reduce risk of aspiration. The goal of the intervention is to achieve the least restrictive liquid diet for each patient. The TWP is designed to reduce liquid viscosity by increasing the amount of liquid mixed with the thickening agent. Patients are seen for weekly feeding therapy with dysphagia management.

Injection Laryngoplasty (IL):

After endotracheal intubation of the patient, a Lindholm laryngoscope is introduced to the vallecula. Vocal cord spreaders are used to retract apart the true and false vocal cords allowing for better visualization of the interarytenoid space. The endotracheal tube is pulled back to the level of the hypopharynx. An endoscopic needle is then used to deliver 0.3-0.6 ccs of hyaluronic acid into the interarytenoid submucosal space. Patients are able to return to normal activity the same day following the procedure. The patient's prior diet is resumed post-operatively and they are scheduled to follow-up in 4 weeks to be reassessed with MBS to determine if TWP can be started. 


\section{Results}

Thirteen patients fit the criteria for the study out of 61 patients identified based on diagnosis of DIN. Their mean age at diagnosis was 21.6 months and 5/13 (38.4\%) were males (Table 3). Common comorbidities included GERD (76.9\%) and asthma/RAD (46.2\%), as well as 3 cases of enteral feeding tubes (23.1\%), 2 cases of congenital heart defects (15.3\%), and one case of Down syndrome (7.7\%). There were no instances of EOE, esophageal stricture, peptic ulcer disease, bronchiolitis, or intrauterine drug exposure. Common medications for comorbid diseases included bronchodilators (69.2\%), inhaled corticosteroids (61.5\%), proton pump inhibitors (PPI) (53.8\%), H-2 receptor antagonists (23.1\%), and Leukotriene receptor antagonists (23.1\%).

All patients received a diagnostic MBS and did not require further evaluation with FEES. Seven patients demonstrated aspiration and 6 patients demonstrated penetration. Of the 7 patients who aspirated, 4 had silent aspiration. Eleven of 13 patients received triple endoscopy (direct laryngoscopy, bronchoscopy, and EGD). One patient underwent just direct laryngoscopy with bronchoscopy because they had little to no gastrointestinal (GI) symptoms. Another patient underwent just direct laryngoscopy with EGD due to few respiratory symptoms.

The overall reduction in thickener level was 3.61 (SD = 1.9), from an average pretreatment level of $5.76(3 / 4$ honey) to a posttreatment level of 2.15 (thin) $(p=0.001)$. Figure 1 illustrates each individual subject's thickener score over the course of therapy. The sign/symptom frequency also reduced by $2.46(S D=4.2)$, from average score of 3.3 to $0.84(p=0.05)$.

The patients were then sub-stratified based on initial DA severity, as determined by their baseline thickener score. Seven patients had moderate dysphagia (baseline MBS of 1-6) and 6 patients had severe dysphagia (baseline MBS of 7-9). The moderate cohort had an overall 2.57 $(S D=1.3)$ reduction in thickener level, while the severe cohort had a $4.83(S D=1.8)$ reduction in thickener level $(p=0.03)$. There was no significant difference between the cohorts in terms of sign/symptom reduction: the moderate cohort reduced by 2.85 points while the severe cohort reduced by 2.0 points $(p=0.88)$. 
Table 3: Patient demographics

\begin{tabular}{|l|c|}
\hline Number of patients & 13 \\
\hline Age at Diagnosis, Months (mean, SD) [R] & $21.6(10.1)[11,47]$ \\
\hline Gender (males, \%) & $5(38.4)$ \\
\hline Ethnicity (N, \%) & $7(53.8)$ \\
Caucasians & $2(15.3)$ \\
African American & $3(23.1)$ \\
Hispanic & $1(7.7)$ \\
Unknown & \\
\hline
\end{tabular}


Figure 1: Thickener levels before and after therapy

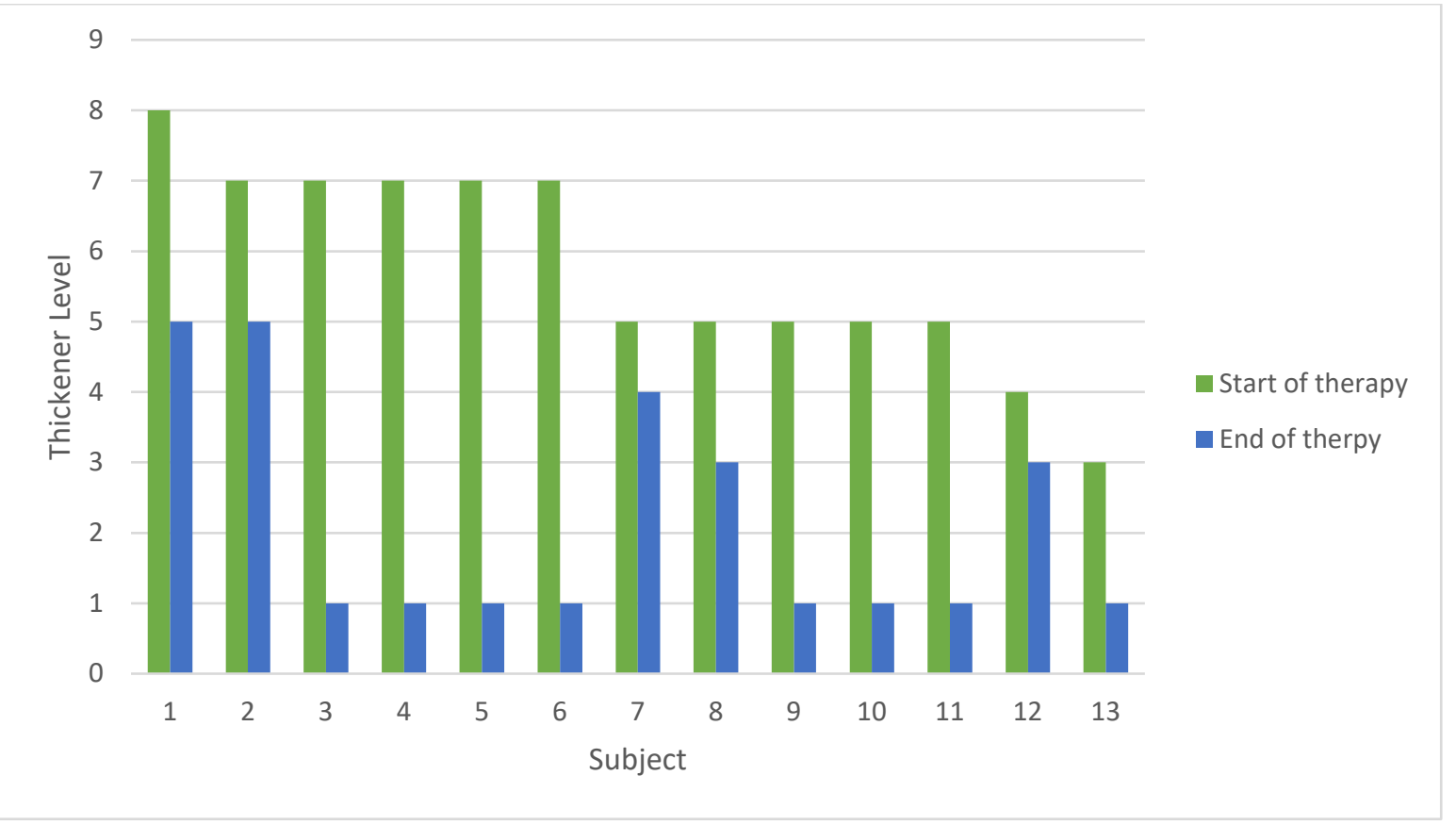




\section{Discussion}

DINs are an emerging topic in aerodigestive disease in pediatrics. While many cohort studies have analyzed LC1s as a whole, further sub-classification has not been performed to isolate the role of DINs in aerodigestive disease. One study on LC1 did mention including DIN patients (described as "low inter-arytenoid height")[5], but no subgroup analysis was performed on that set of patients to draw any meaningful conclusions specifically for DIN patients. A recent review article examined previous studies on IL as treatment option for LC1[6], but none of the published studies delved into the discussion of patient selection and classifying the LC1s further into sub-categories $[15,16,17,18]$. Whether DIN patients were included in these studies is unclear, which left the question of what treatment modalities are suited for DIN patients specifically. The purpose of this study aimed to answer this question.

Patients with laryngeal clefts more often than not have other congenital abnormalities, coincident pathologies, and syndromic associations[11,25]. Our study was unique in that patients with major contributing comorbidities such as neurodevelopmental delay and prior esophageal manipulation were excluded. Of the other less severe comorbidities reported, GERD had a particularly higher incidence among our patients at $76.9 \%$. Previous literature cites the incidence of GERD in LC1 patients anywhere from $21 \%$ to $59 \%[5,26]$. While this may be simply due to sampling error, it can also be explained by the fact that some patients in our cohort were started on PPIs prophylactically rather than due to GERD symptoms. They then continued the medication when EGD revealed mild esophagitis. Nevertheless, GERD remains a significant contributor to these patients' disease process and should be addressed appropriately.

The results of this study show that combination therapy with IL and thickener wean shows promise in young children who present with DA associated with DIN. We observed this through both objective measure via thickener level needed to prevent aspiration/penetration and subjective measure via parent reporting of signs and symptoms. After receiving the dual therapy, patients were able to tolerate thinner liquids which allowed them to reduce their dependence on thickeners, ultimately reducing their DA-related signs/symptoms. These results 
also verify the findings of our previous pilot study, in that DIN patients with more severe dysphagia experience more benefit from IL[27].

Feeding therapy remains integral in the treatment of patients with DINs/LC1s, especially considering how such defects affect all phases of swallowing[9]. Using tolerance to thinner liquid consistencies as an endpoint is not a new concept, and many studies on laryngeal clefts use similar designs for their outcome measure $[17,18,21,22]$. We used a systematic thickener wean to serve both a therapeutic purpose as well as to monitor progress of treatment. Wolter et al. [24] demonstrated thickener weaning to be a successful management technique in most children with DA and predicted that it allowed the swallowing mechanism to steadily adapt to the consistency changes over time. Of note, the scale in table 1 does not represent equal intervals between each numerical score, but was rather designed to obtain measurable information for this study as described in the methods section.

IL is a safe and efficacious treatment option that has emerged more recently. A review of studies utilizing IL for LC1/DIN reported 1 complication from a total of 166 patients[6]. It was noted that there were instances of no improvement with IL, and sometimes slight worsening of symptoms. However, many of the studies included in this review included patients with significant comorbidities which may be influencing the outcome. In our cohort of patients with no significant comorbidities, no complications occurred and all patients experienced clinical improvement. 


\section{Future Directions}

Going forward, there are several ways to use this data to direct future studies, based on our weaknesses. To begin, this study was retrospective in nature and dealt with a small sample size. The limitation to the number of patients resulted from the fact that the current version of TWP was not implemented in PCH until January 2015, so inclusion of prior patients would have deviated from the standardized protocol which was integral to our primary outcome measure. Originally, this study was designed to include a comparative arm with patients treated with TWP alone. Due to system issues related to timely third party authorizations for TWP with a SLP to apply protocol, a prospective study was postponed until infrastructure and system details were secured for flawless TWP post-aerodigestive evaluation. Our group is considering a shift in the clinical paradigm of DA in young children, where DIN is considered a functional defect and therefore the next step is a randomized controlled prospective study of TWP and IL. Furthermore, because no formal description of DIN has been established, the diagnosis remains subjective. At the PCH ADC, 2 otolaryngologists made the diagnosis of DIN based on visual characteristics and palpation of the interarytenoid area which carries subjectivity. Future work could benefit from formal criteria to diagnose DIN and from the development of diagnostic tools to help better direct patient selection. 


\section{Conclusions}

Overall, our findings suggest that treatment of DIN associated DA is possible with a combination of thickeners and IL. This leads to significant clinical improvements of symptoms in young children, along with their ability to tolerate thinner feeds. Although DIN has not been described much in literature, these results suggest that DIN is a contributing factor to the underlying pathology in this subset of DA patients. Future work is necessary to determine whether the benefit from this treatment is truly superior to either surgical or medical treatment alone. 


\section{References}

[1] Arvedson, Joan C. "Assessment of pediatric dysphagia and feeding disorders: clinical and instrumental approaches." Developmental disabilities research reviews 14.2 (2008): 118127.

[2] Benjamin, Bruce, and Andrew Inglis. "Minor congenital laryngeal clefts: diagnosis and classification." Annals of Otology, Rhinology \& Laryngology 98.6 (1989): 417-420.

[3] Smith, Richard JH, Michael B. Neville, and Nancy M. Bauman. "Interarytenoid Notch Height Relative to the Vocal Folds Pilot Study." Annals of Otology, Rhinology \& Laryngology 103.10 (1994): 753-757.

[4] Bakthavachalam, Sivi, James W. Schroeder Jr, and Lauren D. Holinger. "Diagnosis and management of type I posterior laryngeal clefts." Annals of Otology, Rhinology \& Laryngology119.4 (2010): 239-248.

[5] Jefferson, Niall D., Eldar Carmel, and Alan TL Cheng. "Low inter-arytenoid height: a subclassification of type 1 laryngeal cleft diagnosis and management." International journal of pediatric otorhinolaryngology 79.1 (2015): 31-35.

[6] Miglani, Amar, et al. "An Aerodigestive Approach to Laryngeal Clefts and Dysphagia Using Injection Laryngoplasty in Young Children." Current gastroenterology reports 19.12 (2017): 60 .

[7] Rahbar, Reza, et al. "The presentation and management of laryngeal cleft: a 10-year experience." Archives of Otolaryngology-Head \& Neck Surgery 132.12 (2006): 13351341.

[8] Rosenbek, John C., et al. "A penetration-aspiration scale." Dysphagia 11.2 (1996): 93-98.

[9] Strychowsky, Julie E., et al. "Swallowing dysfunction among patients with laryngeal cleft: more than just aspiration?." International journal of pediatric otorhinolaryngology 82 (2016): 38-42. 
[10] Brady, Susan, and Joseph Donzelli. "The modified barium swallow and the functional endoscopic evaluation of swallowing." Otolaryngologic Clinics of North America 46.6 (2013): 1009-1022.

[11] Johnston, Douglas R., et al. "Laryngeal cleft: evaluation and management." International journal of pediatric otorhinolaryngology 78.6 (2014): 905-911.

[12] Farneti, D., and P. Consolmagno. "The Swallowing Centre: rationale for a multidisciplinary management." ACTA otorhinolaryngologica italica 27.4 (2007): 200.

[13] Brown-Whitehorn, Terri F., and Jonathan M. Spergel. "The link between allergies and eosinophilic esophagitis: implications for management strategies." Expert review of clinical immunology 6.1 (2010): 101-109.

[14] Oda, Kayoko, et al. "Dysphagia associated with gastroesophageal reflux disease is improved by proton pump inhibitor." Digestive diseases and sciences 50.10 (2005): 1921-1926.

[15] Cohen, Michael S., et al. "Injection laryngoplasty for type 1 laryngeal cleft in children." Otolaryngology--Head and Neck Surgery 144.5 (2011): 789-793.

[16] Mangat, Harshdeep Singh, and Hamdy El-Hakim. "Injection augmentation of Type 1 laryngeal clefts." Otolaryngology--Head and Neck Surgery 146.5 (2012): 764-768.

[17] Horn, David L., Kim DeMarre, and Sanjay R. Parikh. "Interarytenoid sodium carboxymethylcellulose gel injection for management of pediatric aspiration." Annals of Otology, Rhinology \& Laryngology 123.12 (2014): 852-858.

[18] Thottam, Prasad John, et al. "Outcomes and predictors of surgical management in type 1 laryngeal cleft swallowing dysfunction." The Laryngoscope 126.12 (2016): 2838-2843.

[19] Leonard, Rebecca J., et al. "Effects of bolus rheology on aspiration in patients with dysphagia." Journal of the Academy of Nutrition and Dietetics 114.4 (2014): 590-594.

[20] Yeung, Jeffrey C., et al. "International Pediatric Otolaryngology Group: Consensus guidelines on the diagnosis and management of type I laryngeal clefts." International journal of pediatric otorhinolaryngology 101 (2017): 51-56. 
[21] Chien, Wade, et al. "Type 1 laryngeal cleft: establishing a functional diagnostic and management algorithm." International journal of pediatric otorhinolaryngology 70.12 (2006): 2073-2079.

[22] Ojha, Shilpa, et al. "Type 1 laryngeal cleft: a multidimensional management algorithm." JAMA Otolaryngology-Head \& Neck Surgery 140.1 (2014): 34-40.

[23] National Dysphagia Diet Task Force, and American Dietetic Association. National dysphagia diet: Standardization for optimal care. American Dietetic Associati, 2002.

[24] Wolter, Nikolaus E., et al. "A Systematic Process for Weaning Children With Aspiration From Thickened Fluids." JAMA Otolaryngology-Head \& Neck Surgery 144.1 (2018): 51 56.

[25] Evans, Kathryn L., et al. "Management of posterior laryngeal and laryngotracheoesophageal clefts." Archives of Otolaryngology-Head \& Neck Surgery 121.12 (1995): 1380-1385.

[26] Watters, Karen, Lynne Ferrari, and Reza Rahbar. "Laryngeal cleft." Pediatric Airway Surgery. Vol. 73. Karger Publishers, 2012. 95-100.

[27] Frantz, Garrett. The Role of Injection Laryngoplasty (IL) in Treating Deep Interarytenoid Notch (DIN) associated Dysphagia in Young Children. Diss. The University of Arizona., 2016. 